\title{
Packing spanning graphs from separable families
}

\author{
Asaf Ferber * Choongbum Lee ${ }^{\dagger} \quad$ Frank Mousset ${ }^{\ddagger}$ \\ 18th September 2018
}

\begin{abstract}
Let $\mathcal{G}$ be a separable family of graphs. Then for all positive constants $\epsilon$ and $\Delta$ and for every sufficiently large integer $n$, every sequence $G_{1}, \ldots, G_{t} \in \mathcal{G}$ of graphs of order $n$ and maximum degree at most $\Delta$ such that $e\left(G_{1}\right)+\cdots+e\left(G_{t}\right) \leq(1-\epsilon)\left(\begin{array}{l}n \\ 2\end{array}\right)$ packs into $K_{n}$. This improves results of Böttcher, Hladký, Piguet, and Taraz when $\mathcal{G}$ is the class of trees and of Messuti, Rödl, and Schacht in the case of a general separable family. The result also implies approximate versions of the Oberwolfach problem and of the Tree Packing Conjecture of Gyárfás and Lehel (1976) for the case that all trees have maximum degree at most $\Delta$.

The proof uses the local resilience of random graphs and a special multi-stage packing procedure.
\end{abstract}

\section{Introduction}

A packing of graphs $G_{1}, \ldots, G_{t}$ into a graph $G$ is a collection of graph embeddings $f_{i}: G_{i} \rightarrow G$ whose images $f_{1}\left(G_{1}\right), \ldots, f_{t}\left(G_{t}\right)$ are pairwise edge-disjoint subgraphs of $G$. If such a packing exists, then the graphs $G_{1}, \ldots, G_{t}$ are said to pack into $G$. In this context, the graph $G$ is often called the target graph or the host graph. The packing is said to be perfect if it uses all edges of the host graph, that is, if $e\left(G_{1}\right)+\cdots+e\left(G_{t}\right)=e(G)$.

The problem of finding a perfect packing of identical copies of a graph $H$ in $K_{n}$ has a long and rich history. If $H=K_{k}$, then perfect packings of $H$ into $K_{n}$ are also called block designs or Steiner systems and are a central object of study in the field of design theory. One easily sees that a perfect packing of $K_{k}$

${ }^{*}$ Department of Mathematics, Yale University, New Haven, CT 06520, and Department of Mathematics, MIT, Cambridge, MA 02139-4307. Emails: asaf.ferber@yale.edu, and ferbera@mit.edu.

${ }^{\dagger}$ Department of Mathematics, MIT, Cambridge, MA 02139-4307. Email: cb_lee@math.mit.edu. Research supported by NSF Grant DMS-1362326.

${ }^{\ddagger}$ Department of Computer Science, ETH Zürich, 8092 Zürich, Switzerland. Email: frank.mousset@inf.ethz.ch. Supported by grant no. 6910960 of the Fonds National de la Recherche, Luxembourg. 
into $K_{n}$ can only exist if $(k-1) \mid(n-1)$ and $k(k-1) \mid n(n-1)$. In 1847, Kirkman proved that when $k=3$, then these necessary conditions are also sufficient for the existence of a packing. The conjecture that the conditions are actually sufficient for fixed $k$ and for sufficiently large $n$ is known as the Existence Conjecture for block designs. The 1975 proof of this conjecture by Wilson [26] is considered a breakthrough result in combinatorics. If we move to more general graphs $H$, then once again we see that a perfect packing of identical copies of $H$ into $K_{n}$ cannot exist unless $e(H) \mid\left(\begin{array}{c}n \\ 2\end{array}\right)$ and $\operatorname{gcd}(H) \mid(n-1)$, where the gcd of a graph is the greatest common divisor of the vertex degrees. It is not too hard to come up with examples where these conditions are satisfied, but where there does not exist a perfect packing of $H$ into $K_{n}$; for example one cannot pack two copies of $K_{1,3}$ into $K_{4}$. However, a remarkable theorem of Wilson states that for every $H$ there is some $n_{0}(H)$ such that the divisibility conditions are sufficient whenever $n>n_{0}(H)$ [27]. The number $n_{0}(H)$ is quite large and at present there is little hope of determining what its smallest possible value might be.

This leaves open the question of packing graphs where the number of vertices can be close to $n$. As mentioned above, the obvious divisibility conditions are not always sufficient to guarantee a perfect packing in this case. Clearly, not even two graphs need to pack in general: for example two stars on $2 n$ vertices do not pack into $K_{2 n}$. If we wish to find perfect (or nearly perfect) packings of general graphs, then we need to find some way to exclude these and similar cases. One such way comes in the form of the following beautiful 1976 conjecture of Gyárfas and Lehel [15].

Conjecture 1 (Tree Packing Conjecture). Any collection $T_{1}, \ldots, T_{n}$ of trees such that $v\left(T_{i}\right)=i$ packs into $K_{n}$.

Note that since each $T_{i}$ has exactly $i-1$ edges, the packing of the trees in question would necessarily be perfect. Although Conjecture 1 is still open, there are several partial results. The conjecture is known to be true when the trees satisfy various structural requirements, see [11, 12, 13, 15, 24]. For arbitrary trees, Bollobás [7] proved that one can at least pack the small trees $T_{1}, \ldots, T_{n / \sqrt{2}}$ into $K_{n}$, which can be improved to $\sqrt{3} n / 2$ if the Erdős-Sós conjecture is true. The situation for the larger trees is much worse: it is only known that the five trees $T_{n-4}, T_{n-3}, T_{n-2}, T_{n-1}, T_{n}$ pack into $K_{n}$ [28] (the previously best known result for three trees $T_{n-2}, T_{n-1}, T_{n}$ was proved in [16]), although Balogh and Palmer [4] proved that one can pack the $n^{1 / 4} / 10$ largest trees into $K_{n+1}$ (note that an extra vertex is needed), and they also proved that one can pack the $n^{1 / 3} / 4$ largest trees into $K_{n}$ if each has maximum degree at least $2 n^{2 / 3}$. In any case, packing even a small linear fraction of the large trees appears to be a very hard problem.

The problem becomes more tractable if one additionally assumes that each tree $T_{i}$ has bounded maximum degree. A well-known result of Böttcher, 
Hladký, Piguet, and Taraz [8] shows that in this setting, one can achieve nearly perfect packings of trees, provided that the size of the trees is bounded away from $n$ :

Theorem 1.1 (Böttcher, Hladký, Piguet, and Taraz [8]). For all positive constants $\epsilon$ and $\Delta$ and for every sufficiently large integer $n$, the following holds. Every collection $T_{1}, \ldots, T_{t}$ of trees of order at most $(1-\epsilon) n$ and maximum degree at most $\Delta$ such that $e\left(T_{1}\right)+\cdots+e\left(T_{t}\right) \leq(1-\epsilon)\left(\begin{array}{l}n \\ 2\end{array}\right)$ packs into $K_{n}$.

In particular, their theorem yields the following asymptotic version of the Tree Packing Conjecture.

Corollary 1.2. For all positive constants $\epsilon$ and $\Delta$ and for every sufficiently large integer $n$, every sequence $T_{1}, \ldots, T_{(1-\epsilon) n}$ of trees with $v\left(T_{i}\right)=i$ and maximum degree at most $\Delta$ packs into $K_{n}$.

The fact that the graphs $T_{i}$ from Conjecture 1 are assumed to be trees is very important (otherwise the conjecture is simply not true). However, there is no reason why Theorem 1.1 should hold only for trees. In fact, we believe that Theorem 1.1 remains true if one replaces the word "trees" by "graphs". While this is not known, a result similar to Theorem 1.1 holds at least for all separable families of graphs. In the literature, a family $\mathcal{G}$ of graphs is typically said to be separable if it satisfies a separator theorem, meaning there exist constants $c, \alpha<1$ such that the removal of $O\left(v(G)^{\alpha}\right)$ vertices from any $G \in \mathcal{G}$ can partition the graph into disjoint subgraphs of size at most $c \cdot v(G)$. However, following [22, we adopt a slightly more general definition:

Definition 1.3 (separable). A graph $G$ is $(\delta, K)$-separable if by removing $\delta v(G)$ vertices from $G$ one can obtain a graph in which every component has size at most $K$. A family $\mathcal{G}$ of graphs is separable if for every $\delta>0$ there is some $K$ such that each graph $G \in \mathcal{G}$ is $(\delta, K)$-separable.

As shown in [22, every family of graphs that is separable in the familiar sense given above is also separable according to Definition [1.3. It is worth mentioning that by the famous separator theorem of Alon, Seymour and Thomas [1], all non-trivial minor-closed families of graphs (such as planar graphs, graphs of bounded treewidth, etc.) are separable. In particular, the class of trees is separable. Messuti, Rödl, and Schacht proved the following generalisation of Theorem 1.1 to separable families of graphs [22].

Theorem 1.4 (Messuti, Rödl, and Schacht [22]). Assume that $\mathcal{G}$ is a separable family of graphs. Then for all positive constants $\epsilon$ and $\Delta$ and for every sufficiently large integer $n$, the following holds. Every collection $G_{1}, \ldots, G_{t} \in \mathcal{G}$ of $t \leq(1-\epsilon) n$ graphs of order at most $(1-\epsilon) n$ and maximum degree at most $\Delta$ such that $e\left(G_{1}\right)+\cdots+e\left(G_{t}\right) \leq(1-\epsilon)\left(\begin{array}{l}n \\ 2\end{array}\right)$ packs into $K_{n}$. 
Note that in Theorems 1.1 and 1.4, the graphs being packed have a number of vertices which is bounded away from $n$. This situation is not untypical: in graph embedding problems, it is almost always easier to embed a nearly spanning structure than it is to embed a spanning structure. However, in light of the fact that the hard part of Conjecture 1 is the embedding of the very large trees, it is highly desirable to develop methods that allow the packing of spanning subgraphs. The contribution of this paper is the following stronger version of Theorem 1.4.

Theorem 1.5. Assume that $\mathcal{G}$ is a separable family of graphs. Then for all positive constants $\epsilon$ and $\Delta$, there exists $n_{0}=n_{0}(\epsilon, \Delta, \mathcal{G})$ such that the following holds for all $n \geq n_{0}$. Every collection $G_{1}, \ldots, G_{t} \in \mathcal{G}$ of graphs of order at most $n$ and maximum degree at most $\Delta$ such that $e\left(G_{1}\right)+\cdots+e\left(G_{t}\right) \leq(1-\epsilon)\left(\begin{array}{l}n \\ 2\end{array}\right)$ packs into $K_{n}$.

While the theorem applies to all separable families, the result is new even when $\mathcal{G}$ is the family of trees. From it, one also immediately obtains a stronger approximate version of the Tree Packing Conjecture:

Corollary 1.6. For all positive constants $\epsilon$ and $\Delta$ and for every sufficiently large integer $n$, every collection $T_{\epsilon n}, \ldots, T_{n}$ of trees with $v\left(T_{i}\right)=i$ and maximum degree at most $\Delta$ packs into $K_{n}$.

Since the family of all 2-regular graphs is separable, Theorem 1.5 also yields an approximate version of the 'Oberwolfach problem' (proposed by Ringel in 1967) which states that given any 2-regular graph $F$ on $n$ vertices, where $n$ is odd, there exists a perfect packing of copies of $F$ into $K_{n}$. Bryant and Scharaschkin [9] proved that the exact statement of the Oberwolfach problem is correct for infinitely many values of $n$.

There are many possible ways in which Theorem 1.5 could be strengthened. Most obviously, the restriction that the graphs are from a separable family is likely unnecessary; the theorem as stated should hold for any boundeddegree graphs $G_{1}, \ldots, G_{t}$. Secondly, one would like to know the dependence of $n_{0}$ on the parameters $\epsilon$ and $\Delta$. It is not clear what kind of dependence follows from our proof, but it is certainly very far from optimal. Thirdly, it would be interesting to have an efficient algorithm which, given the graphs $G_{1}, \ldots, G_{t}$, produces a packing into $K_{n}$. Our proof does not give such an algorithm, although we pretend (without proof) that if $\mathcal{G}$ is the family of trees, then a variation of our argument yields a randomized algorithm which packs the given trees with high probability and which runs in time $O_{\epsilon, \Delta}\left(n^{c}\right)$ for some reasonable absolute constant $c>0$. Lastly, there is some interest in the problem of packing graphs of bounded maximum degree into random and pseudorandom graphs. Our proof of Theorem 1.5 can be modified with little effort to show that for any constant $p \in(0,1]$, a.a.s. the given graphs $G_{1}, \ldots, G_{t}$ pack into the random graph $G(n, p)$ (see below for the definitions), 
provided that $e\left(G_{1}\right)+\cdots+e\left(G_{t}\right) \leq(1-\epsilon) p\left(\begin{array}{l}n \\ 2\end{array}\right)$. In its current form, our proof does not provide packings into any model of pseudorandom graphs.

Very recently, Kim, Kühn, Osthus, and Tyomkyn [18] have managed to address the first and last points mentioned above: the condition of separability in Theorem 1.5 is not necessary - and in fact the graphs do not just pack into $K_{n}$ but into any dense quasi-random graph.

Before continuing, we give some further pointers to the literature. Recently, Keevash extended the results of Wilson on perfect packings of $K_{k}$ to quasi-random hypergraphs [17]. Barber, Kühn, Lo, and Osthus studied perfect packings of triangles or even cycles into graphs of large minimum degree [6]. Packings of arbitrary small graphs into graphs of large minimum degree were considered by Glock, Kühn, Lo, Montgomery, and Osthus in [14. A wellknown conjecture of Kelly states that every regular tournament on $n$ vertices can be decomposed into $(n-1) / 2$ directed Hamilton cycles. The proof of a strong form of this conjecture for large $n$ was given by Kühn and Osthus [19]; the method used seems to be widely applicable, see [20].

\section{Notation}

We use $[n]$ for the set $\{1,2, \ldots, n\}$. If $G$ is a graph and $X$ is a subset of the vertices, then $N_{G}(X)$ denotes the neighbourhood of $X$ in $G$, i.e., the set of all vertices adjacent to a vertex of $X$. We define $G-X$ as the subgraph of $G$ induced on $V(G) \backslash X$.

The disjoint union of two graphs $G$ and $H$ is written as $G+H$. If $G$ is a graph and $n$ is an integer, then we write $G \times n$ for the graph consisting of $n$ disjoint copies of $G$.

If $f: G \rightarrow H$ is a graph embedding, then $\operatorname{Im} f$ denotes the image of $f$ (considered as a subset of $V(H)$ ) and $\overline{\operatorname{Im}} f$ denotes the complement $V(H) \backslash \operatorname{Im} f$ of the image. On the other hand, we write $f(G)$ to denote the subgraph of $H$ that $G$ is mapped to (which is always isomorphic to $G$ ).

We use two closely related random graph models. $G(n, p)$ denotes the random graph on $n$ vertices in which every edge is present independently with probability $p . B(n, p)$ denotes the random bipartite graph with parts of size $n$ in which every edge that goes between the parts is present independently with probability $p$.

A sequence of events $A_{n}$ is said to occur asymptotically almost surely (a.a.s.) if $\mathbf{P}\left[A_{n}\right] \rightarrow 1$ as $n \rightarrow \infty$. By a common abuse of language, we simply say that the event $A_{n}$ occurs a.a.s..

\section{Preliminaries}

For the proof we need some probabilistic tools. First of all, we use the wellknown Chernoff bounds [21, Theorem 2.1]. 
Lemma 2.1 (Chernoff). Assume that $X_{1}, \ldots, X_{n}$ are jointly independent binary random variables such that $\mathbf{P}\left[X_{i}=1\right]=p$ and $\mathbf{P}\left[X_{i}=0\right]=1-p$. Let $X=X_{1}+\cdots+X_{n}$. Then for any $\epsilon \in(0,1)$

$$
\mathbf{P}[|X-\mathbf{E}[X]| \geq \epsilon n p] \leq 2 e^{-\epsilon^{2} n p / 3} .
$$

Additionally, we use the following variant of the Azuma-Hoeffding inequality. The formulation below follows by applying the usual Azuma-Hoeffding inequality [10, Theorem 27] to the supermartingale $Y_{1}, \ldots, Y_{n}$ defined by $Y_{k}:=$ $\sum_{i=1}^{k}\left(X_{i}-\mu_{i}\right)$.

Lemma 2.2 (Azuma-Hoeffding). Let $X_{1}, \ldots, X_{n}$ be random variables where $0 \leq X_{i} \leq c_{i}$ for all $i$ and where there are real numbers $\mu_{1}, \ldots, \mu_{n}$ such that

$$
\mathbf{E}\left[X_{i} \mid X_{i-1}, \ldots, X_{1}\right] \leq \mu_{i}
$$

holds for all $i$. Let $X=X_{1}+\cdots+X_{n}$ and $\mu=\mu_{1}+\cdots+\mu_{n}$. Then for any $\epsilon>0$

$$
\mathbf{P}[X \geq(1+\epsilon) \mu] \leq e^{-\epsilon^{2} \mu^{2} /\left(2 \sum_{i=1}^{n} c_{i}^{2}\right)} .
$$

In our proof, the following notion will be very important:

Definition 2.3 (Local resilience). Let $\mathcal{P}$ be any monotone increasing graph property. The local resilience of a graph $G$ with respect to $\mathcal{P}$ is the minimum number $r$ such that by deleting at most $r$ edges at each vertex $v$ of $G$, one can obtain a graph not in $\mathcal{P}$.

We will only need to consider the case where $\mathcal{P}$ is the property of containing a perfect matching. For this, we have the following lemma, whose proof we omit (it is almost identical to the proof of Theorem 2.3 in the seminal paper of Sudakov and $\mathrm{Vu}[25]$ ). We remark that although the lemma gives a local resilience of almost $1 / 2$, for our purposes, any positive constant would suffice.

Lemma 2.4. The following holds for every constant $\epsilon>0$ and every function $p=p(n)=\omega(\log n / n)$. With probability $1-o\left(n^{-1}\right)$ the local resilience of the random bipartite graph $B(n, p)$ with respect to the property of containing a perfect matching is greater than $(1-\epsilon) n p / 2$.

The following lemma (a special case of a result of Bárány and Doerr [5]) will turn out to be surprisingly useful.

Lemma 2.5 (Bárány and Doerr). Let $d$ be a positive integer. For every finite set $A \subseteq[0,1]^{d}$ and every positive integer $m$, there is a partition of $A$ into $m$ parts $A_{1}, \ldots, A_{m}$ such that for all $i \in[m]$

$$
\sum_{a \in A_{i}} a \in \frac{1}{m} \sum_{a \in A} a+3 d[-1,1]^{d} .
$$


Finally, we shall need a result on packing nearly spanning graphs with small components into random graphs. For brevity we will adopt the following definition.

Definition 2.6. Let $\mathcal{B C}(n, K)$ denote the family of all graphs of order at most $n$ in which every component has size at most $K$.

The next lemma sates that graphs from $\mathcal{B C}((1-\epsilon) n, K)$ pack asymptotically optimally into dense random graphs on $n$ vertices, and moreover, this packing can be chosen to be random-like in the sense that fixed subsets of the graphs are spread evenly over the vertices of $G(n, p)$. Since the proof is rather involved, we postpone it to Section 4. As a side remark, this lemma is the only part of our proof that is not algorithmic (in the sense that its proof does not suggest an efficient randomized algorithm that produces the desired packing with high probability). However, if all the components are trees, then one can use the method of [3] to obtain an algorithmic proof.

Lemma 2.7. Let $\epsilon, \alpha, \beta, K>0$ and $p \in(0,1]$ be constants and let $G \sim G(n, p)$. Assume that $G_{1}, \ldots, G_{t}$ are graphs in $\mathcal{B C}((1-\epsilon) n, K)$ such that

$$
e\left(G_{1}\right)+\cdots+e\left(G_{t}\right) \leq(1-\epsilon)\left(\begin{array}{l}
n \\
2
\end{array}\right) p
$$

where each graph $G_{i}$ has at least $(1-2 \epsilon) n$ vertices. Moreover, assume that $A_{1}, \ldots, A_{t}$ and $B_{1}, \ldots, B_{t}$ are sets where for all $i$ we have $A_{i}, B_{i} \subseteq V\left(G_{i}\right)$, $\left|A_{i}\right| \leq \alpha n$, and $\left|B_{i}\right| \leq \beta n$. Then a.a.s. there exists a packing $\left\{f_{i}: G_{i} \rightarrow G\right\}$ of the graphs $G_{1}, \ldots, G_{t}$ into $G$ with the following properties:

(1) for every vertex $v$ of $G$ there are at most $2 \alpha t$ values $i \in[t]$ such that $v \in f_{i}\left(A_{i}\right)$ and at most $4(\beta+\epsilon) t$ values $i \in[t]$ such that $v \in f_{i}\left(B_{i}\right) \cup \overline{\operatorname{Im}} f_{i}$;

(2) for any two distinct vertices $u, v$ of $G$ there are at most $5(\beta+\epsilon)^{2} t$ values $i \in[t]$ such that $\{u, v\} \subseteq f_{i}\left(B_{i}\right) \cup \overline{\operatorname{Im}} f_{i}$.

\section{Proof of Theorem 1.5}

Let $\mathcal{G}$ be a separable family of graphs and let positive constants $\epsilon$ and $\Delta$ be given. Additionally, fix constants $\gamma, \delta, \zeta, p_{0}, K$ such that

$$
0<K^{-1} \ll \delta \ll \zeta \ll \gamma \ll p_{0} \ll \epsilon,
$$

where the notation $a \ll b$ means that $a$ should be sufficiently small depending on $b$. We assume throughout that $n$ is a sufficiently large integer.

Let $G_{1}, \ldots, G_{t} \in \mathcal{G}$ be graphs of order at most $n$ and maximum degree at most $\Delta$ such that $e\left(G_{1}\right)+\cdots+e\left(G_{t}\right) \leq(1-\epsilon)\left(\begin{array}{l}n \\ 2\end{array}\right)$. The goal is to pack these graphs into $K_{n}$. Note that by combining two graphs into one if they have fewer 
than $n / 2$ non-isolated vertices, we can assume without loss of generality that at most one graph $G_{i}$ has less than $n / 4$ edges, which implies that $t \leq 2 n$. By adding isolated vertices, we can also assume that each graph $G_{i}$ has exactly $n$ vertices.

Since $\mathcal{G}$ is a separable family, each graph $G_{i}$ contains a set $S_{i} \subseteq V\left(G_{i}\right)$ of size $\delta n$ such that $G_{i}-S_{i}$ only has components of size at most $K$ (note that we will consistently omit rounding brackets throughout the proof). For each graph $G_{i}$ we now fix such a set $S_{i}$.

An independent set $I$ in a graph $G$ is said to be 2-independent if any two vertices of $I$ have disjoint neighbourhoods in $G$. Since each $G_{i}$ has maximum degree at most $\Delta$, we can greedily find a 2-independent set $I_{i}$ of size $\gamma n$ in $G_{i}$ that is disjoint from $S_{i}$.

The general idea of the proof is to proceed in three phases: in the first phase we pack all the graphs $G_{i}-S_{i}-I_{i}$, in the second phase we extend this packing to a packing of the graphs $G_{i}-I_{i}$, and in the final phase, we extend that packing to a packing of the graphs $G_{i}$.

\section{Preparations}

Let $M:=\gamma^{-2}$ and fix a collection $Z^{(1)}, \ldots, Z^{(M)}$ of $M$ disjoint subsets of $V\left(K_{n}\right)$ containing $\zeta n$ vertices each (this is possible since $M \zeta n \leq n$ ).

Now let $p:=\gamma^{2}\left(1-p_{0}\right)$ and split $K_{n}$ into $M+1$ edge-disjoint random subgraphs $\Gamma^{(0)}, \ldots, \Gamma^{(M)}$ as follows. First, let $\left\{X_{e} \mid e \in E\left(K_{n}\right)\right\}$ be jointly independent random variables distributed uniformly on the interval $[0,1]$. Then we define $\Gamma^{(0)}$ to be the subgraph of $K_{n}$ with the edge set $\left\{e \mid X_{e} \in\left[0, p_{0}\right]\right\}$, and for $k \in[M]$ we define $\Gamma^{(k)}$ to be the subgraph with edge set $\left\{e \mid X_{e} \in\left(p_{0}+(k-1) p, p_{0}+k p\right]\right\}$.

The graphs $\Gamma^{(0)}, \Gamma^{(1)}, \ldots, \Gamma^{(M)}$ partition $K_{n}$ into edge-disjoint subgraphs. Moreover, one can check that $\Gamma^{(0)}$ is distributed as $G\left(n, p_{0}\right)$ and that the graphs $\Gamma^{(1)}, \ldots, \Gamma^{(M)}$ are distributed as $G(n, p)$.

Similarly, we will split our collection of graphs $G_{1}, \ldots, G_{t}$ into $M$ groups as follows. For each $i \in[t]$ define the vector $a_{i}:=\left(1, \frac{2 e\left(G_{i}\right)}{\Delta n}\right) \in[0,1]^{2}$, and let $A:=\left\{a_{1}, \ldots, a_{t}\right\}$. By applying Lemma 2.5 to $A$ we obtain a partition

$$
A:=\bigcup_{1 \leq k \leq M} A_{k}
$$

for which

$$
\sum_{a \in A_{k}} a \in \frac{1}{M} \sum_{a \in A} a \pm 6[-1,1]^{2} .
$$

Clearly, since the first coordinate of each $a_{i}$ is 1 , it follows that $\left|A_{k}\right| \leq \frac{t}{M}+6$ for all $k$. Moreover, since the second coordinate of each $a_{i}$ is $\frac{2 e\left(G_{i}\right)}{\Delta n}$, it follows that

$$
\sum_{i: a_{i} \in A_{k}} \frac{e\left(G_{i}\right)}{\Delta n} \in \frac{\sum_{i} e\left(G_{i}\right)}{M \Delta n} \pm 6 .
$$


By setting $\mathcal{B}^{(k)}:=\left\{i \in[t] \mid a_{i} \in A_{k}\right\}$ we obtain a partition

$$
\{1, \ldots, t\}=\bigcup_{1 \leq k \leq M} \mathcal{B}^{(k)}
$$

which satisfies $\max _{k}\left|\mathcal{B}^{(k)}\right| \leq \frac{t}{M}+6 \leq 3 \gamma^{2} n$ and

$$
\sum_{i \in \mathcal{B}^{(k)}} e\left(G_{i}\right) \leq \frac{e\left(G_{1}\right)+\cdots+e\left(G_{t}\right)}{M}+6 \Delta n \leq(1-\epsilon / 2)\left(\begin{array}{l}
n \\
2
\end{array}\right) p,
$$

where we used that $e\left(G_{1}\right)+\cdots+e\left(G_{t}\right) \leq(1-\epsilon)\left(\begin{array}{c}n \\ 2\end{array}\right)$.

Phase I: packing the $G_{i}-I_{i}-S_{i}$

For each $k \in[M]$, we will pack the collection $\left\{G_{i}-I_{i}-S_{i} \mid i \in \mathcal{B}^{(k)}\right\}$ into the graph $\Gamma^{(k)}-Z^{(k)}$. The edges of $\Gamma^{(k)}$ that are incident with $Z^{(k)}$ will only be used in the second phase. We also note that this leaves the graph $\Gamma^{(0)}$ completely untouched; the edges of $\Gamma^{(0)}$ are reserved for the final phase.

Our goal is to apply Lemma 2.7. Let us first set everything up. Since $Z^{(k)}$ was fixed beforehand, we can see that $\Gamma^{(k)}-Z^{(k)}$ is a random graph distributed as $G((1-\zeta) n, p)$. As $\zeta \ll \gamma$ there is some $\xi>0$ so that

$$
1-\delta-\gamma=(1-\xi)(1-\zeta)
$$

In fact, as $\zeta, \delta \ll \gamma$, we have (say) $\gamma / 2 \leq \xi \leq 2 \gamma$. By the definition of $S_{i}$ and using $v\left(G_{i}-I_{i}-S_{i}\right)=(1-\delta-\gamma) n$, we then have $G_{i}-I_{i}-S_{i} \in$ $\mathcal{B} C((1-\xi)(1-\zeta) n, K)$ and $v\left(G_{i}-I_{i}-S_{i}\right) \geq(1-2 \xi)(1-\zeta) n$. Let $A_{i}$ be the set of neighbours of $S_{i}$ in $G_{i}-I_{i}-S_{i}$ and let $B_{i}$ be the set of neighbours of $I_{i}$ in $G_{i}-I_{i}-S_{i}$. Note that $\left|A_{i}\right| \leq \Delta\left|S_{i}\right| \leq \Delta \delta n$ and $\left|B_{i}\right| \leq \Delta\left|I_{i}\right| \leq \Delta \gamma n$.

Now we can apply Lemma 2.7 and obtain for each $k \in[M]$ a packing

$$
\left\{f_{i}: G_{i}-I_{i}-S_{i} \rightarrow \Gamma^{(k)}-Z^{(k)} \mid i \in \mathcal{B}^{(k)}\right\}
$$

with the following properties (using $\max _{k}\left|\mathcal{B}^{(k)}\right| \leq 3 \gamma^{2} n$ ):

(P1) for every vertex $v$ of $\Gamma^{(k)}-Z^{(k)}$ there are at most $6 \Delta \delta \gamma^{2} n$ values $i \in \mathcal{B}^{(k)}$ such that $v \in f_{i}\left(A_{i}\right)$ and at most $12(\Delta \gamma+\xi) \gamma^{2} n \leq 50 \Delta \gamma^{3} n$ values $i \in \mathcal{B}^{(k)}$ such that $v \in f_{i}\left(B_{i}\right) \cup\left(V\left(K_{n}\right) \backslash\left(Z^{(k)} \cup \operatorname{Im} f_{i}\right)\right)$;

(P2) for any two distinct vertices $u, v$, of $\Gamma^{(k)}-Z^{(k)}$ there are at most $15(\Delta \gamma+$ $\xi)^{2} \gamma^{2} n \leq 200 \Delta^{2} \gamma^{4} n$ values $i \in \mathcal{B}^{(k)}$ such that $\{u, v\} \subseteq f_{i}\left(B_{i}\right) \cup\left(V\left(K_{n}\right) \backslash\right.$ $\left.\left(Z^{(k)} \cup \operatorname{Im} f_{i}\right)\right)$. 


\section{Phase II: extending to a packing of the $G_{i}-I_{i}$}

The next step is to extend the embeddings $f_{i}: G_{i}-I_{i}-S_{i} \rightarrow \Gamma^{(k)}-Z^{(k)}$ constructed above to edge-disjoint embeddings $g_{i}: G_{i}-I_{i} \rightarrow \Gamma^{(k)}$. For this, we will use essentially the same greedy procedure that was used in the proof of Theorem 1.4 [22]. Fix some $k \in[M]$. For each $i \in \mathcal{B}^{(k)}$, we will embed $S_{i}$ into $Z^{(k)}$ using the edges of $\Gamma^{(k)}$.

First, observe that by the Chernoff bound (Lemma 2.1) and the union bound, a.a.s. every set of at most $\Delta$ vertices of $\Gamma^{(k)}$ has $(1+o(1))\left|Z^{(k)}\right| p^{\Delta} \geq$ $\zeta \gamma^{2 \Delta} n / 2$ common neighbours in $Z^{(k)}$. From now on, we assume that this is the case.

Now we process the values $i \in \mathcal{B}^{(k)}$ one by one. Fix some $i$ and assume for an induction that we already have edge-disjoint embeddings $\left\{g_{j}: G_{j}-I_{j} \rightarrow\right.$ $\left.\Gamma^{(k)} \mid j \in \mathcal{B}^{(k)} \cap[i-1]\right\}$ with the property that for each vertex $v$, there are at most $\zeta^{2} n$ values $j \in \mathcal{B}^{(k)} \cap[i-1]$ such that $v \in g_{j}\left(S_{j}\right)$. We show that we can also embed $S_{i}$ to obtain a packing of $\left\{G_{j}-I_{j} \mid j \in \mathcal{B}^{(k)} \cap[i]\right\}$ which does not violate this property.

Order the vertices of $S_{i}$ as $v_{1}, \ldots, v_{\delta n}$. Note that the neighbours of $S_{i}$ in $G_{i}-S_{i}-I_{i}$ are already embedded by $f_{i}$, and it only remains to embed the vertices in $S_{i}$. We embed these vertices one by one, where we need to embed each vertex into the common neighborhood of the images of the neighbours that we have embedded up to that point. When doing this we never want to use edges that have been used by an embedding of one of the graphs $G_{j}-I_{j}$ where $j<i$.

Suppose that we have embedded $v_{1}, \ldots, v_{\ell-1}$ and want to embed $v_{\ell}$. Let $N_{\ell}$ be the set of the neighbours of $v_{\ell}$ that have been embedded up to that point and let $\tilde{N}_{\ell}$ be the image of this set in $\Gamma^{(k)}$. Since $N_{\ell}$ contains at most $\Delta$ vertices, we know that $\tilde{N}_{\ell}$ has at least $\zeta \gamma^{2 \Delta} n / 2$ common neighbours in $Z^{(k)}$ (by the observation above). We need to embed $v_{\ell}$ into a common neighbour $u$ of $\tilde{N}_{\ell}$ in $Z^{(k)}$ that satisfies the following three properties: (i) none of the edges between $\tilde{N}_{\ell}$ and $u$ has been used by an embedding of a graph $G_{j}-I_{j}$ where $j \in \mathcal{B}^{(k)} \cap[i-1]$; (ii) $u$ is not the image of a vertex $v_{1}, \ldots, v_{\ell-1}$; (iii) there are at most $\zeta^{2} n-1$ values $j \in \mathcal{B}^{(k)} \cap[i-1]$ such that $u \in f_{j}\left(S_{j}\right)$. If such a common neighbour $u$ exists, then we can embed $v_{\ell}$ to $u$ and continue with the next vertex.

We now show that such a vertex exists. Firstly, by (P1) and by the induction hypothesis, and using $\zeta \ll \gamma$, there exist at least $\zeta \gamma^{2 \Delta} n / 4$ common neighbours of $\tilde{N}_{\ell}$ that satisfy (i). Moreover, since $\ell \leq \delta n \leq \zeta \gamma^{2 \Delta} / 8$, among these there are at least $\zeta \gamma^{2 \Delta} n / 8$ that also satisfy (ii). Finally, the total number of vertices in $Z^{(k)}$ used by previous embeddings is at most $\delta n \cdot\left|\mathcal{B}^{(k)}\right| \leq \delta n^{2}$ (counted with multiplicities), which is not enough to cover each of those $\zeta \gamma^{2 \Delta} n / 8$ vertices $\zeta^{2} n$ times (as $\delta \ll \zeta \ll \gamma$ ). So there must be a common neighbour satisfying (i), (ii), and (iii), which can be the image of $v_{\ell}$.

This procedure results in packings $\left\{g_{i}: G_{i}-I_{i} \rightarrow \Gamma^{(k)} \mid i \in \mathcal{B}^{(k)}\right\}$, one for 
each $k \in[M]$. The union of these packings is a packing

$$
\left\{g_{i}: G_{i}-I_{i} \rightarrow K_{n} \backslash \Gamma^{(0)} \mid i \in[t]\right\} .
$$

We finish this subsection by stating the crucial property that will allow us to complete the packing of the graphs $G_{i}-I_{i}$ to a packing of the graphs $G_{i}$ :

Claim 3.1. For every vertex $v$ of $K_{n}$, there are at most $\gamma^{0.9} n$ values $i \in[t]$ such that

$$
v \in g_{i}\left(N_{G_{i}}\left(I_{i}\right)\right) \cup \overline{\operatorname{Im}} g_{i} .
$$

For any two distinct vertices $u, v$ of $K_{n}$, there are at most $\gamma^{1.9} n$ values $i \in[t]$ such that

$$
\{u, v\} \subseteq g_{i}\left(N_{G_{i}}\left(I_{i}\right)\right) \cup \overline{\operatorname{Im}} g_{i} .
$$

Proof. Let $F_{i}:=g_{i}\left(N_{G_{i}}\left(I_{i}\right)\right) \cup \overline{\operatorname{Im}} g_{i}$. For a fixed $k \in[M]$ and any vertex $v \in V\left(K_{n}\right) \backslash Z^{(k)}$, by $(\mathrm{P} 1)$ there are at most $50 \Delta^{3} \gamma^{3} n$ values $i \in \mathcal{B}^{(k)}$ such that $v \in F_{i}$. In total these cases contribute at most $50 \Delta^{3} \gamma n$ values of $i$. On the other hand, for any $v$, there is at most one $k \in[M]$ such that $v \in Z^{(k)}$ (as the $Z^{(k)}$ are disjoint sets), which contributes at $\operatorname{most}^{\max _{k}}\left|\mathcal{B}^{(k)}\right| \leq 3 \gamma^{2} n$ values of $i$. This gives the first statement. The second statement follows along the same lines, using (P2) instead of (P1).

\section{Auxiliary graphs and local resilience}

Now that we have a packing $\left\{g_{i}: G_{i}-I_{i} \rightarrow K_{n} \backslash \Gamma^{(0)} \mid i \in[t]\right\}$, we need to complete it into a packing $\left\{h_{i}: G_{i} \rightarrow K_{n} \mid i \in[t]\right\}$. For this we will only use edges of $\Gamma^{(0)}$. However, since the graphs are spanning, we cannot embed vertices one by one as we did in Phase II. Luckily, the sets $I_{i}$ have a much nicer structure than the sets $S_{i}$ : they are 2 -independent sets. This will allow us to use a method based on perfect matchings in bipartite graphs to embed these sets.

For each $i \in[t]$, we define an auxiliary bipartite graph $B_{i}$ as follows:

- the parts of $B_{i}$ are $\left\{g_{i}\left(N_{G_{i}}(v)\right) \mid v \in I_{i}\right\}$ and $\overline{\operatorname{Im}} g_{i}$ (note that these are both sets of size $\gamma n$ );

- there is an edge between $X$ and $x$ if $x$ lies in the common neighbourhood of $X$ in $\Gamma^{(0)}$.

Note that since $I_{i}$ is 2-independent, the sets $g_{i}\left(N_{G_{i}}(v)\right)$ are disjoint for all $v \in I_{i}$. Observe also that it is possible to extend $g_{i}$ to an embedding $h_{i}: G_{i} \rightarrow K_{n}$ if and only if $B_{i}$ contains a perfect matching: that is, if $x$ is matched to $X=g_{i}\left(N_{G_{i}}(v)\right)$ then we can embed $v$ as $x$. Since our goal is to extend the embeddings in an "edge-disjoint way", we actually need to claim that $B_{i}$ is "robust" in some sense with respect to the property of containing a perfect matching. For this we use the notion of local resilience and show: 
Claim 3.2. The local resilience of $B_{i}$ with respect to containing a perfect matching is a.a.s. greater than $\gamma^{1.1} n$ for every $i \in[t]$.

Proof. Fix some $k \in[M]$. We start the proof with an observation about the joint distribution of the pair $\left(\Gamma^{(0)}, \Gamma^{(k)}\right)$. Recall that $\Gamma^{(k)}$ is the graph of edges $e \in E\left(K_{n}\right)$ for which the uniform random variable $X_{e} \in[0,1]$ takes a value in the interval $\left(p_{0}+(k-1) p, p_{0}+k p\right]$. Thus the marginal distribution of $\Gamma^{(k)}$ is $G(n, p)$. Now suppose we condition on the value of $\Gamma^{(k)}$, that is, we expose the set of edges $e$ for which $X_{e} \in\left(p_{0}+(k-1) p, p_{0}+k p\right]$. Then, by symmetry, the remaining values $X_{e}$ for $e \notin E\left(\Gamma^{(k)}\right)$ are independent random variables distributed uniformly in the set $\left[0, p_{0}+(k-1) p\right] \cup\left(p_{0}+k p, 1\right]$. By definition, $\Gamma^{(0)}$ contains exactly those edges $e \in E\left(K_{n}\right)$ for which $X_{e} \in\left[0, p_{0}\right]$. Thus each edge $e \notin E\left(\Gamma^{(k)}\right)$ is an edge of $\Gamma^{(0)}$ independently and with probability $p_{0} /(1-p)$. In other words, the conditional distribution of $\Gamma^{(0)}$ given $\Gamma^{(k)}$ is $G\left(n, p_{0} /(1-p)\right) \backslash \Gamma^{(k)}$. This shows that to generate the pair $\left(\Gamma^{(0)}, \Gamma^{(k)}\right)$, we may proceed as follows: first sample a random graph $\Gamma^{(k)} \sim G(n, p)$, then sample a second random graph $\tilde{\Gamma}^{(0)} \sim G\left(n, p_{0} /(1-p)\right)$ independently of $\Gamma^{(k)}$, and finally let $\Gamma^{(0)}=\tilde{\Gamma}^{(0)} \backslash \Gamma^{(k)}$. This observation will be important in the argument below.

Additionally, for each possible outcome of $\Gamma^{(k)}$, we can fix a canonical packing $\left\{g_{i}: G_{i}-I_{i} \rightarrow \Gamma^{(k)} \mid i \in \mathcal{B}^{(k)}\right\}$ that depends only on $\Gamma^{(k)}$ (provided such a packing exists; although it does a.a.s.).

With this in mind, we can generate the auxiliary graph $B_{i}$ for $i \in \mathcal{B}^{(k)}$ as follows. First expose the edges of $\Gamma^{(k)}$. By the canonical choice of the packing, this completely determines the embedding $g_{i}: G_{i}-I_{i} \rightarrow \Gamma^{(k)}$. Next, sample an independent random graph $\tilde{\Gamma}^{(0)} \sim G\left(n, p_{0} /(1-p)\right)$ as above. Let $\tilde{B}_{i}$ be defined like $B_{i}$ except that we put an edge between $X$ and $x$ if $x$ is a common neighbour of $X$ in $\tilde{\Gamma}^{(0)}$ (instead of $\left.\Gamma^{(0)}\right)$. Finally, let $B_{i}$ be obtained from $\tilde{B}_{i}$ by removing the edges $(X, x)$ where there exists $y \in X$ such that $\{y, x\} \in E\left(\Gamma^{(k)}\right)$. Since $\Gamma^{(0)}=\tilde{\Gamma}^{(0)} \backslash \Gamma^{(k)}$, this definition of $B_{i}$ results in the same distribution as the one given at the beginning of this subsection.

Because $I_{i}$ is a 2-independent set and because $\tilde{\Gamma}^{(0)}$ is independent of $\Gamma^{(k)}$ (hence independent of the sets $g_{i}\left(N_{G_{i}}(v)\right)$ for $\left.v \in S_{i}\right)$, we see that $\tilde{B}_{i}$ is distributed as a random bipartite graph $B\left(\gamma n, p_{0}^{\Delta} /(1-p)^{\Delta}\right)$. So by Lemma 2.4. with probability $1-o\left(n^{-1}\right)$ the local resilience of $\tilde{B}_{i}$ with respect to containing a perfect matching is greater than $\gamma n p_{0}^{\Delta} / 2 \geq 2 \gamma^{1.1} n$.

Finally, $B_{i}$ is obtained from $\tilde{B}_{i}$ by removing at most $\Delta \cdot \Delta\left(\Gamma^{(k)}\right)$ edges at each vertex. However, by the Chernoff bound (Lemma 2.1) and the union bound, the maximum degree of $\Gamma^{(k)}$ is a.a.s. at most $2 n p \leq 2 \gamma^{2} n$ for each $k \in$ $[M]$. So even after deleting these edges, the local resilience of $B_{i}$ with respect to containing a perfect matchings is still greater than $2 \gamma^{1.1} n-2 \Delta \gamma^{2} n \geq \gamma^{1.1} n$. The claim follows by an application of the union bound over all $i$. 


\section{Phase III: extending to a packing of the $G_{i}$}

We now complete the packing $\left\{g_{i}: G_{i}-I_{i} \rightarrow K_{n} \mid i \in[t]\right\}$ to a packing $\left\{h_{i}: G_{i} \rightarrow K_{n} \mid i \in[t]\right\}$ according to the following randomized procedure. We process the auxiliary graphs $B_{1}, \ldots, B_{t}$ one by one. Given $i \in[t]$ let us say that a perfect matching in $B_{i}$ is eligible if it does not contain an edge $(X, x)$ such that one of the embeddings $h_{1}, \ldots, h_{i-1}$ uses an edge $\{y, x\}$ where $y \in X$. Fix a collection of $\gamma^{1.2} n$ edge-disjoint eligible perfect matchings in $B_{i}$, assuming such a collection exists; if not, the procedure fails. Extend $g_{i}$ to $h_{i}$ by choosing a perfect matching from this collection uniformly at random.

If it succeeds, this procedure produces a collection of edge-disjoint embeddings $g_{i}: G_{i} \rightarrow K_{n}$ for $i \in[t]$. We only need to show that the algorithm succeeds with positive probability. This follows from the following claim, which completes the proof since $t=O(n)$.

Claim 3.3. For each $i$ the following holds: the probability that the algorithm fails in step $i$ given that it did not fail in any previous step is at most $o(1 / n)$.

Proof. For $j \in[t]$, let $F_{j}:=g_{i}\left(N_{G_{j}}\left(I_{j}\right)\right) \cup \overline{\operatorname{Im}} g_{j}$ and recall that $\left|F_{j}\right| \leq(\Delta+1) \gamma n$.

Fix $i$ and suppose that we construct $h_{1}, \ldots, h_{i-1}$ according to the randomized procedure described above. Say that an edge $\{u, v\}$ is forbidden if it is used by one of the embeddings $h_{1}, \ldots, h_{i-1}$. Then it is enough to show that for each $v \in F_{i}$ there are at most $\gamma^{1.1} n /(2 \Delta)$ vertices $u \in F_{i} \backslash\{v\}$ such that $\{u, v\}$ is an edge of $\Gamma^{(0)}$ that is forbidden. Indeed, this implies that at every vertex of $B_{i}$, there are at most $\gamma^{1.1} n / 2$ non-eligible edges, which by the local resilience (Claim 3.2) implies that there is a collection of at least $\gamma^{1.1} n / 2 \geq \gamma^{1.2} n$ edge-disjoint perfect matchings.

Fix some vertex $v \in F_{i}$. First of all, note that an edge $\{u, v\} \in E\left(\Gamma^{(0)}\right)$ can only be used by the embedding $h_{j}$ if $\{u, v\} \subseteq F_{j}$. By the first statement of Claim 3.1, there are at most $\gamma^{0.9} n$ values $j<i$ such that $v \in F_{j}$. Additionally, for all but at most $\gamma^{1.2} n$ values $j<i$ such that $v \in F_{j}$, we have and $\left|F_{i} \cap F_{j}\right| \leq$ $\gamma^{1.5} n$. Indeed, otherwise there would be some $u \in F_{i}$ such that $\{u, v\}$ is contained in $\gamma^{2.7} n^{2} /\left|F_{i}\right| \geq \gamma^{1.7} n /(\Delta+1)$ many sets $F_{j}$, contradicting the second statement of Claim 3.1

From the $\gamma^{1.2} n$ values for which this fails, we get at most $\Delta \gamma^{1.2} n \leq \gamma^{1.1} n / 4$ forbidden edges incident to $v$. For the at most $\gamma^{0.9} n$ remaining ones, recall that $h_{j}$ was obtained by choosing a perfect matching uniformly at random from a set of $\gamma^{1.2} n$ edge-disjoint perfect matchings. So the probability that $h_{j}$ uses an edge from $v$ to a vertex $u \in F_{i}$ is at most

$$
\frac{\left|F_{i} \cap F_{j}\right|}{\gamma^{1.2} n} \leq \gamma^{0.3}
$$

independently of all previous choices. Since the maximum degree of each graph $G_{j}$ is at most $\Delta$, the Azuma-Hoeffding inequality (Lemma 2.2) implies that 
with probability

$$
1-e^{-\frac{\gamma^{2 \cdot 4_{n}}}{2 \Delta^{2}}}=1-o\left(n^{-2}\right),
$$

there are at most $2 \Delta \gamma^{1.2} n \leq \gamma^{1.1} n /(2 \Delta)$ forbidden edges in $\Gamma^{(0)}\left[F_{i}\right]$ incident to $v$. The claim now follows by taking the union bound over all vertices $v$.

\section{Proof of Lemma 2.7}

Before we can prove Lemma 2.7, we need to collect some auxiliary results. Recall that $K_{\ell} \times b$ denotes the graph consisting of $b$ disjoint copies of $K_{\ell}$. Firstly, we will need the following result, which states that there exist asymptotically optimal packings of copies of $K_{\ell} \times(1-o(1)) n / \ell$ in $G(n, p)$.

Lemma 4.1. Let $\epsilon, p \in(0,1)$ be constants and let $\ell \in \mathbb{N}$ be sufficiently large. Let $N=(1-\epsilon) \frac{n p}{\ell-1}$ and let $b=(1-\epsilon) \frac{n}{\ell}$. Then a graph $G \sim G(n, p)$ a.a.s. contains a family of edge-disjoint subgraphs $C_{1}, \ldots, C_{N}$ where each graph $C_{i}$ is isomorphic to $K_{\ell} \times b$ and where for each vertex $v$ of $G$ there are at least $(1-\epsilon) N$ values $s \in[N]$ such that $v \in V\left(C_{s}\right)$.

We note that the lemma actually holds for values of $p$ as low as polylog $n$. $n^{-(\ell-2) /\left(\left(\begin{array}{l}\ell \\ 2\end{array}\right)-1\right)}$, with much the same proof.

The proof of Lemma 4.1 uses a very powerful result of Pippenger and Spencer [23. Recall that the chromatic index $\chi^{\prime}(\mathcal{H})$ of a hypergraph $\mathcal{H}$ is defined as the smallest number $c$ such that there exists a colouring of the edges of $\mathcal{H}$ with $c$ colours in which every colour class forms a matching (i.e., a set of independent edges). For a hypergraph $\mathcal{H}$, let $\delta(\mathcal{H})$ be the minimum degree of $\mathcal{H}$, let $\Delta(\mathcal{H})$ be the maximum degree of $\mathcal{H}$, and let $\Delta_{2}(\mathcal{H})$ be the maximum co-degree of any two distinct vertices in $\mathcal{H}$. Then clearly $\chi^{\prime}(\mathcal{H}) \geq \Delta(\mathcal{H})$. Pippenger and Spencer proved that if $\delta(\mathcal{H}) \sim \Delta(\mathcal{H})$ and $\Delta_{2}(\mathcal{H})=o(\Delta(\mathcal{H})$ ), then this lower bound is asymptotically tight:

Theorem 4.2 (Pippenger and Spencer [23]). For every $k \geq 2$ and $\delta>0$, there exist $\delta^{\prime}>0$ and $n_{0}$ such that if $\mathcal{H}$ is a k-uniform hypergraph on $n \geq n_{0}$ vertices satisfying

$$
\delta(\mathcal{H}) \geq\left(1-\delta^{\prime}\right) \Delta(\mathcal{H}) \quad \text { and } \quad \Delta_{2}(\mathcal{H}) \leq \delta^{\prime} \Delta(\mathcal{H})
$$

then

$$
\chi^{\prime}(\mathcal{H}) \leq(1+\delta) \Delta(H) .
$$

We will also use the following simple fact about the distribution of cliques in sufficiently dense random graphs. We omit the proof (it is a simple application of the Chernoff bound). 
Lemma 4.3. The following holds for every integer constant $\ell \geq 3$ and every $p=p(n) \geq(\log n)^{-3}$. With probability $1-o\left(n^{-1}\right)$ every edge of $G(n, p)$ is contained in $(1 \pm o(1))\left(\begin{array}{c}n \\ \ell-2\end{array}\right) p^{\left(\begin{array}{l}\ell \\ 2\end{array}\right)-1}$ copies of $K_{\ell}$ and any two distinct edges of $G(n, p)$ are contained in $o\left(n^{\ell-2} p^{\left(\begin{array}{l}\ell \\ 2\end{array}\right)-1}\right)$ copies of $K_{\ell}$. Similarly, with probability $1-o\left(n^{-1}\right)$, every vertex of $G(n, p)$ is contained in $(1 \pm o(1))\left(\begin{array}{c}n \\ \ell-1\end{array}\right) p^{\left(\begin{array}{l}\ell \\ 2\end{array}\right)}$ copies of $K_{\ell}$.

Proof of Lemma 4.1. Let us fix constants $0<\zeta \ll \delta \ll \epsilon$ and let $G \sim G(n, p)$. We first show that $G$ a.a.s. contains a large collection of edge-disjoint copies of $K_{\ell}$ such that each vertex is contained in at least $(1-\zeta) n p /(\ell-1)$ copies.

For this, colour each edge of $G$ with a colour chosen uniformly at random from $\{1, \ldots, \log n\}$ and write $G_{i}$ for the graph of edges in colour $i$. Then $G_{i} \sim G(n, q)$ where $q=p /(\log n)^{2} \geq(\log n)^{-3}$. By Lemma 2.1 (the Chernoff bound) and the union bound, a.a.s. the maximum degree of each $G_{i}$ is at most $(1+o(1)) n q$. By Lemma 4.3 and the union bound, a.a.s. each graph $G_{i}$ has the following additional properties:

(i) each vertex is contained in at least $(1-o(1))\left(\begin{array}{c}n \\ \ell-1\end{array}\right) q^{\left(\begin{array}{l}\ell \\ 2\end{array}\right)}$ copies of $K_{\ell}$;

(ii) each edge is contained in $(1 \pm o(1))\left(\begin{array}{c}n \\ \ell-2\end{array}\right) q^{\left(\begin{array}{l}\ell \\ 2\end{array}\right)-1}$ copies of $K_{\ell}$;

(iii) any pair of distinct edges is contained in $o\left(n^{\ell-2} q^{\left(\begin{array}{l}\ell \\ 2\end{array}\right)-1}\right)$ copies of $K_{\ell}$.

For each $i$, consider the $\left(\begin{array}{l}\ell \\ 2\end{array}\right)$-uniform hypergraph $\mathcal{H}_{i}$ whose vertices are the edges of $G_{i}$ and whose edges are the edge sets of copies of $K_{\ell}$ in $G_{i}$. Note that a matching in $\mathcal{H}_{i}$ corresponds to a collection of edge-disjoint copies of $K_{\ell}$ in $G_{i}$. By (ii) and (iii), we have

$$
(1-o(1)) \Delta\left(\mathcal{H}_{i}\right) \leq\left(\begin{array}{c}
n \\
\ell-2
\end{array}\right) q^{\left(\begin{array}{c}
\ell \\
2
\end{array}\right)-1} \leq(1+o(1)) \delta\left(\mathcal{H}_{i}\right)
$$

and $\Delta_{2}\left(\mathcal{H}_{i}\right)=o\left(\Delta\left(\mathcal{H}_{i}\right)\right)$ for all $i$. Thus by Theorem 4.2 the edges of each hypergraph $\mathcal{H}_{i}$ can be coloured with $\chi^{\prime}\left(\mathcal{H}_{i}\right)=(1+o(1))\left(\begin{array}{c}n \\ \ell-2\end{array}\right) q^{\left(\begin{array}{c}\ell \\ 2\end{array}\right)-1}$ colours such that each colour class is a matching. Fix such a colouring for each $\mathcal{H}_{i}$.

For a given vertex $v$ and a given colour class $M$ in the colouring of $\mathcal{H}_{i}$, let us write $v \cap M$ for the set of (hyper)edges in $M$ that contain an edge of $G_{i}$ incident to $v$. Since $M$ corresponds to a collection of edge-disjoint copies of $K_{\ell}$ in $G_{i}$, the set $v \cap M$ can also be seen as the set of the copies of $K_{\ell}$ in $M$ that contain $v$. Because $G_{i}$ has maximum degree at most $(1+o(1)) n q$ we trivially have $|v \cap M| \leq(1+o(1)) n q /(\ell-1)$ for any colour class $M$. On the other hand, there are fewer than $\zeta \cdot \chi^{\prime}\left(\mathcal{H}_{i}\right)$ colour classes $M$ in the colouring of $\mathcal{H}_{i}$ such that $|v \cap M| \leq(1-\zeta) n q /(\ell-1)$ : otherwise we would have $\sum_{M}|v \cap M| \leq\left(1-\zeta^{2}+o(1)\right)\left(\begin{array}{c}n \\ \ell-1\end{array}\right) q^{\left(\begin{array}{l}\ell \\ 2\end{array}\right)}$ (where the sum is over colour classes $M$ in the colouring of $\mathcal{H}_{i}$ ), contradicting (i). 
We now construct a collection $\mathcal{C}_{i}$ of edge-disjoint copies of $K_{\ell}$ in $G_{i}$ as follows: choose a colour class $M_{i}$ in the colouring of $\mathcal{H}_{i}$ uniformly at random and let $\mathcal{C}_{i}$ be the copies of $K_{\ell}$ corresponding to the edges in $M_{i}$. Note that this random choice is done independently for each $i$. Thus, by the above considerations, the probability that $\left|v \cap M_{i}\right| \leq(1-\zeta) n q /(\ell-1)$ is at most $\zeta-$ independently of what happens for other $i$ ! Thus, if we let $\mathcal{C}:=\mathcal{C}_{1} \cup \cdots \cup \mathcal{C}_{\log n}$, then by the Chernoff bound (Lemma 2.1) and the union bound, a.a.s. every vertex $v$ is contained in at least $(1-2 \zeta) n p /(\ell-1)$ copies of $K_{\ell}$ in $\mathcal{C}$.

Now that we have a collection $\mathcal{C}$ of many edge-disjoint copies of $K_{\ell}$, we use Theorem 4.2 a second time to find many copies of $K_{\ell} \times b$ in this collection. This time, let $\mathcal{H}$ be the hypergraph on $V(G)$ whose edges are the vertex sets of the copies of $K_{\ell}$ in $\mathcal{C}$. By the property of $\mathcal{C}$ obtained above, we have $\delta(\mathcal{H}) \geq(1-2 \zeta) n p /(\ell-1)$. On the other hand, as the maximum degree of $G$ is at most $(1+o(1)) n p$, we have $\Delta(\mathcal{H}) \leq(1+o(1)) n p /(\ell-1)$. Finally, we have $\Delta_{2}(\mathcal{H}) \leq 1$. By Theorem 4.2, there is a proper edge colouring of $\mathcal{H}$ with at most $(1+\delta) n p /(\ell-1)$ colours. Each colour class corresponds to a disjoint union of copies of $K_{\ell}$ from $\mathcal{C}$.

Since each vertex is contained in at least $(1-2 \zeta) n p /(\ell-1) \geq(1+\epsilon / 3) N$ copies of $K_{\ell}$ in $\mathcal{C}$, there must be at least this many colour classes. The proof is completed by observing that all but at most $\delta n p /(\ell-1) \leq \epsilon N / 3$ colour classes must have size at least $(1-\delta) n / \ell \geq b$. Indeed, by the Handshaking Lemma, $\mathcal{C}$ contains at least $(1-2 \zeta) \frac{n^{2} p}{\ell(\ell-1)}$ copies of $K_{\ell}$, and if $\delta n p /(\ell-1)$ colour classes would have size below $(1-\delta) n / \ell$, then there would be fewer than

$$
\delta(1-\delta) \frac{n^{2} p}{\ell(\ell-1)}+(1-\delta)(1+o(1)) \frac{n^{2} p}{\ell(\ell-1)} \leq(1-2 \zeta) \frac{n^{2} p}{\ell(\ell-1)}
$$

copies. Thus there exist at least $N$ edge-disjoint copies of $K_{\ell} \times b$. Since for each vertex $v$, there are at most $(2 \zeta+\delta) n p /(\ell-1) \leq \epsilon N$ colour classes that do not contain $v$, this also gives the second part of the claim,

Our next lemma is about packing graphs from $\mathcal{B C}((1-\epsilon) \ell, K)$ into $K_{\ell}$. This is made easy for us since we can simply use Theorem 1.4. However, more direct arguments also work; see for example [2].

Lemma 4.4. For all constants $\epsilon \in(0,1)$ and $K>0$ there exists $\ell_{0}$ such that the following holds for all $\ell \geq \ell_{0}$. Any graphs $G_{1}, \ldots, G_{t} \in \mathcal{B C}((1-\epsilon) \ell, K)$ such that $e\left(G_{1}\right)+\cdots+e\left(G_{t}\right) \leq(1-\epsilon)\left(\begin{array}{l}\ell \\ 2\end{array}\right)$ pack into $K_{\ell}$.

Proof. We can assume that $\epsilon$ is sufficiently small. Let $C=C(\epsilon)$ be a sufficiently large constant. Note that if $t \leq(1-\epsilon) \ell$, then the lemma follows immediately from Theorem 1.4 .

The idea is to transform the collection $G_{1}, \ldots, G_{t}$ into a smaller collection $\tilde{G}_{1}, \ldots, \tilde{G}_{s}$ with the following properties: (1) at most four graphs $\tilde{G}_{i}$ have fewer than $(1-\epsilon) 3 \ell / 4$ edges; (2) each graph $\tilde{G}_{i}$ is an edge-disjoint union of one or 
more unique graphs $G_{j}$ (here unique means that the assignment of graphs $G_{j}$ to graphs $\tilde{G}_{i}$ is a function); (3) $\tilde{G}_{i}$ has at most $(1-\epsilon / 2) \ell$ vertices; (4) the maximum size of a component in $\tilde{G}_{i}$ is at most $C K$.

Suppose that we can do this. Then by (2) we have

$$
e\left(\tilde{G}_{1}\right)+\cdots+e\left(\tilde{G}_{s}\right)=e\left(G_{1}\right)+\cdots+e\left(G_{t}\right) \leq(1-\epsilon)\left(\begin{array}{l}
\ell \\
2
\end{array}\right)
$$

Together with (1) this implies easily that $s \leq(1-\epsilon) \ell$. Now by (3-4) and Theorem 1.4, the graphs $\tilde{G}_{1}, \ldots, \tilde{G}_{s}$ pack into $K_{\ell}$ (assuming that $\ell$ is large enough). By (2) this corresponds to a packing of the graphs $G_{1}, \ldots, G_{t}$ and we are done. It remains to show how to obtain $\tilde{G}_{1}, \ldots, \tilde{G}_{s}$.

For this we use the following procedure, which operates in two phases. Let $\tilde{G}_{1}, \ldots, \tilde{G}_{s}$ denote the current collection, where initially $s=t$ and $\tilde{G}_{i}=G_{i}$. By adding isolated vertices, assume also that $v\left(\tilde{G}_{i}\right)=(1-\epsilon) \ell$ for each $i$.

In the first phase, we repeat the following as long as there are at least two graphs $\tilde{G}_{i}$ and $\tilde{G}_{j}$ with at most $(1-\epsilon) \ell / 4$ edges. Given two such graphs, we note that they both have at least $(1-\epsilon) \ell / 2$ isolated vertices. Therefore, by taking their disjoint union and removing isolated vertices, we obtain a graph on at most $(1-\epsilon) \ell$ vertices. This new graph still has maximum component size at most $K$.

We end up with a collection $\tilde{G}_{1}, \ldots, \tilde{G}_{s}$ in which at most one graph has at most $(1-\epsilon) \ell / 4$ edges. In the second phase, as long as there are at least three graphs $\tilde{G}_{i}, \tilde{G}_{j}, \tilde{G}_{k}$ that have strictly more than $(1-\epsilon) \ell / 4$ and at most $(1-\epsilon) 3 \ell / 4$ edges, we replace these three graphs by a single graph as follows.

Let $r:=(1-\epsilon / 2) \ell /(C K)$. If $C$ is sufficiently large, then we can greedily group the components of $\tilde{G}_{i}$ (all of which have size bounded by $K$ ) into graphs $H_{i 1}, \ldots, H_{i r}$ where each graph contains at most $(1-\epsilon / 4) C K$ vertices. Similarly, we obtain graphs $H_{j 1}, \ldots, H_{j r}$ and $H_{k 1}, \ldots, H_{k r}$ for $\tilde{G}_{j}$ and $\tilde{G}_{k}$. If $C$ is large enough, then for each $m \in[r]$ there exists a graph $H_{m}$ on at most $C K$ vertices that is the edge-disjoint union of $H_{i m}, H_{j m}, H_{k m}$ (for this one can for example use Theorem 1.4, although much simpler arguments also work). Then we replace $\tilde{G}_{i}, \tilde{G}_{j}, \tilde{G}_{k}$ by the disjoint union $H:=H_{1}+\ldots+H_{r}$. Note that $H$ has strictly more than $(1-\epsilon) 3 \ell / 4$ edges, so it will not be touched again; moreover, $v(H) \leq(1-\epsilon / 2) \ell$ and every component in $H$ has size at most $C K$. This completes the proof of the lemma.

We can now prove Lemma 2.7 .

Lemma 2.7. Let $\epsilon, \alpha, \beta, K>0$ and $p \in(0,1]$ be constants and let $G \sim G(n, p)$. Assume that $G_{1}, \ldots, G_{t}$ are graphs in $\mathcal{B C}((1-\epsilon) n, K)$ such that

$$
e\left(G_{1}\right)+\cdots+e\left(G_{t}\right) \leq(1-\epsilon)\left(\begin{array}{l}
n \\
2
\end{array}\right) p,
$$

where each graph $G_{i}$ has at least $(1-2 \epsilon) n$ vertices. Moreover, assume that $A_{1}, \ldots, A_{t}$ and $B_{1}, \ldots, B_{t}$ are sets where for all $i$ we have $A_{i}, B_{i} \subseteq V\left(G_{i}\right)$, 
$\left|A_{i}\right| \leq \alpha n$, and $\left|B_{i}\right| \leq \beta n$. Then a.a.s. there exists a packing $\left\{f_{i}: G_{i} \rightarrow G\right\}$ of the graphs $G_{1}, \ldots, G_{t}$ into $G$ with the following properties:

(1) for every vertex $v$ of $G$ there are at most $2 \alpha t$ values $i \in[t]$ such that $v \in f_{i}\left(A_{i}\right)$ and at most $4(\beta+\epsilon)$ t values $i \in[t]$ such that $v \in f_{i}\left(B_{i}\right) \cup \overline{\operatorname{Im}} f_{i}$;

(2) for any two distinct vertices $u, v$ of $G$ there are at most $5(\beta+\epsilon)^{2} t$ values $i \in[t]$ such that $\{u, v\} \subseteq f_{i}\left(B_{i}\right) \cup \overline{\operatorname{Im}} f_{i}$.

Proof. Let $\ell$ be a sufficiently large constant and let $\delta>0$ be a sufficiently small constant. Let $N=(1-\delta) \frac{n p}{\ell-1}$ and $b=(1-\delta) \frac{n}{\ell}$. By Lemma 4.1, $G$ a.a.s. contains a collection of $N$ edge-disjoint copies of $K_{\ell} \times b$ such that each vertex is contained in at least $(1-\delta) N$ copies. Let us denote by

$$
F_{s}=F_{s 1}+\cdots+F_{s b} \subseteq G
$$

the $s$-th copy of $K_{\ell} \times b$ in this collection, where each graph $F_{s j}$ is a copy of $K_{\ell}$.

By applying Lemma 2.5 to the sequence of vectors $\left(1, \frac{e\left(G_{i}\right)}{K n}\right) \in[0,1]^{2}$, there exists a partition

$$
\{1, \ldots, t\}=\mathcal{B}_{1} \cup \cdots \cup \mathcal{B}_{N}
$$

which satisfies $\left|\mathcal{B}_{s}\right|=t / N \pm 6$ and

$$
\sum_{i \in \mathcal{B}_{s}} e\left(G_{i}\right)=\frac{e\left(G_{1}\right)+\cdots+e\left(G_{t}\right)}{N} \pm 6 K n \leq(1-\epsilon / 2) \frac{n(\ell-1)}{2}
$$

for all $s$, where the upper bound holds if $\ell$ is sufficiently large.

The idea is to pack all the graphs $G_{i}$ for $i \in \mathcal{B}_{s}$ into the graph $F_{s}$. Since $F_{s}$ is isomorphic to $K_{\ell} \times b$, we want to write each such $G_{i}$ as a disjoint union of subgraphs of graphs $H_{i 1}, \ldots, H_{i b}$. With this in mind, fix some $i \in[t]$ and let $\mathcal{C}_{i}$ be the set of components of $G_{i}$. With each component $C \in \mathcal{C}_{i}$ we associate a vector

$$
v_{C}=\left(\frac{v(C)}{K}, \frac{e(C)}{K^{2}}, \frac{\left|V(C) \cap A_{i}\right|}{K}, \frac{\left|V(C) \cap B_{i}\right|}{K}\right) \in[0,1]^{4} .
$$

By Lemma 2.5 there exists a partition of $\mathcal{C}_{i}$ into $b$ sets $\mathcal{C}_{i 1}, \ldots, \mathcal{C}_{i b}$ such that for each $j \in[b]$ we have

$$
\sum_{C \in \mathcal{C}_{i j}} v_{C} \in \frac{1}{b}\left(\frac{v\left(G_{i}\right)}{K}, \frac{e\left(G_{i}\right)}{K^{2}}, \frac{\left|A_{i}\right|}{K}, \frac{\left|B_{i}\right|}{K}\right)+12[-1,1]^{4} .
$$

Note that by letting $H_{i j}$ be the subgraph containing exatly the components $C \in \mathcal{C}_{i j}$, this partition corresponds to a way of writing $G_{i}$ as a disjoint union

$$
G_{i}=H_{i 1}+\cdots+H_{i b}
$$

of graphs satisfying the following properties, where the upper bound in each case follows from the definition of $b$, the fact that $\ell$ is a sufficiently large constant, and from the bounds $\left|A_{i}\right| \leq \alpha n$ and $\left|B_{i}\right| \leq \beta n$ : 
(i) $v\left(H_{i j}\right)=\frac{v(G)}{b} \pm 12 K \in \ell-[5 / 2,1 / 2] \cdot \epsilon \ell$;

(ii) $e\left(H_{i j}\right)=\frac{e\left(G_{i}\right)}{b} \pm 12 K^{2} \leq(1+\epsilon / 2) e\left(G_{i}\right) \ell / n$;

(iii) $\left|V\left(H_{i j}\right) \cap A_{i}\right|=\frac{\left|A_{i}\right|}{b} \pm 12 K \leq 1.5 \alpha \ell$;

(iv) $\left|V\left(H_{i j}\right) \cap B_{i}\right|=\frac{\left|B_{i}\right|}{b} \pm 12 K \leq 1.5 \beta \ell$.

Using Lemma 4.4 it is now easy to see that for each $s$ and $j$, the family $\left\{H_{i j} \mid i \in \mathcal{B}_{s}\right\}$ packs into $F_{s j}$ (which is isomorphic to $K_{\ell}$ ): the condition on the number of vertices is (i), the condition on the number of edges follows from (2) and (ii), and each component of $H_{i j}$ has size at most $K$.

Therefore we can fix edge-disjoint embeddings $\left\{f_{i j}: H_{i j} \rightarrow F_{s j} \mid i \in \mathcal{B}_{s}\right\}$. For each $s$ and $j$, let $\sigma_{s j}$ be an automorphism of $F_{s j}$ chosen independently and uniformly at random among the $\ell$ ! choices (which simply amounts to a random permutation of the vertices of $F_{s j}$ ). Clearly if for $i \in \mathcal{B}_{s}$ we set $g_{i j}:=\sigma_{s j} \circ f_{i j}$ then each set $\left\{g_{i j}: H_{i j} \rightarrow F_{s j} \mid i \in \mathcal{B}_{s}\right\}$ is also a packing. For each $i$, let $g_{i}: G_{i} \rightarrow G$ be the embedding obtained by combining the partial embeddings $g_{i 1}, \ldots, g_{i b}$. Then the collection $\left\{g_{i} \mid i \in[t]\right\}$ is a packing of the graphs $G_{i}$ into $G$.

Since each vertex is contained in at least $(1-\delta) N$ graphs $F_{s}$, and since the embeddings in each graph $F_{s j}$ are random, it is easy to check (using the Azuma-Hoeffding and union bounds) that (1) and (2) are a.a.s. satisfied for all vertices.

\section{Acknowledgements}

We would like to thank Rajko Nenadov for useful discussions in the early stages of this project.

\section{Bibliography}

[1] N. Alon, P. Seymour, and R. Thomas, A separator theorem for nonplanar graphs, Journal of the American Mathematical Society 3 (1990), no. 4, 801-808.

[2] N. Alon and R. Yuster, Every $H$-decomposition of $K_{n}$ has a nearly resolvable alternative, European Journal of Combinatorics 21 (2000), no. 7, 839-845.

[3] D. Bal, A. Frieze, M. Krivelevich, P. Loh, Packing Tree Factors in Random and Pseudo-Random Graphs, Electronic Journal of Combinatorics 21 (2014), no. 2, paper \#P2.8. 
[4] J. Balogh and C. Palmer, On the Tree Packing Conjecture, SIAM Journal on Discrete Mathematics 27 (2013), no. 4, 1995-2006.

[5] I. Bárány and B. Doerr, Balanced partitions of vector sequences, Linear Algebra and its Applications 414 (2006), no. 2-3, 464-469.

[6] B. Barber, D. Kühn, A. Lo, and D. Osthus, Edge-decompositions of graphs with high minimum degree, Advances in Mathematics 288 (2016), 337385.

[7] B. Bollobás, Some remarks on packing trees, Discrete Mathematics 46 (1983), no. 2, 203-204.

[8] J. Böttcher, J. Hladký, D. Piguet, and A. Taraz, An approximate version of the tree packing conjecture, Israel Journal of Mathematics, to appear.

[9] D. Bryant and V. Scharaschkin Complete solutions to the Oberwolfach problem for an infinite set of orders, Journal of Combinatorial Theory, Series B 99 (2009), no. 6, 904-918.

[10] F. Chung and L. Lu, Concentration inequalities and martingale inequalities: a survey, Internet Mathematics 3 (2006), no. 1, 79-127.

[11] E. Dobson, Packing almost stars into the complete graph, Journal of Graph Theory 25 (1997), no. 2, 169-172.

[12] E. Dobson, Packing trees into the complete graph, Combinatorics, Probability \& Computing 11 (2002), no. 3, 263-272.

[13] E. Dobson, Packing trees of bounded diameter into the complete graph, Australasian Journal of Combinatorics 37 (2007), 89-100.

[14] S. Glock, D. Kühn, A. Lo, R. Montgomery, and D. Osthus, On the decomposition threshold of a given graph, arXiv:1603.04724 [math.CO] (2016).

[15] A. Gyárfás and J. Lehel, Packing trees of different order into $K_{n}$, Colloq. Math. Soc. János Bolyai 18, Combinatorics (1978), 463-469.

[16] A. M. Hobbs, B. A. Bourgeois, and J. Kasiraj, Packing trees in complete graphs, Discrete Mathematics 67 (1987), no. 1, 27-42.

[17] P. Keevash, The existence of designs, arXiv:1401.3665 [math.c0] (2014).

[18] J. Kim, D. Kühn, D. Osthus, and M. Tyomkyn, A blow-up lemma for approximate decompositions, arXiv:1604.07282 [math.CO] (2016).

[19] D. Kühn and D. Osthus, Hamilton decompositions of regular expanders: a proof of Kelly's conjecture for large tournaments, Advances in Mathematics 237 (2013), 62-146. 
[20] D. Kühn and D. Osthus, Hamilton decompositions of regular expanders: applications, Journal of Combinatorial Theory, Series B 104 (2014), 1-27.

[21] C. McDiarmid, Concentration, Probabilistic Methods for Algorithmic Discrete Mathematics (1998).

[22] S. Messuti, V. Rödl, and M. Schacht, Packing minor-closed families of graphs into complete graphs, submitted.

[23] N. Pippenger and J. Spencer, Asymptotic behavior of the chromatic index for hypergraphs, Journal of Combinatorial Theory, Series A 51 (1989), no. $1,24-42$.

[24] Y. Roditty, Packing and covering of the complete graph. III. On the tree packing conjecture. Scientia, Series A: Mathematical Sciences 1 (1988), 81-85.

[25] B. Sudakov and V. Vu, Local resilience of graphs, Random Structures \& Algorithms 33 (2008), no. 4, 409-433.

[26] R. M. Wilson, An existence theory for pairwise balanced designs, III: Proof of the existence conjectures, Journal of Combinatorial Theory, Series A 18 (1975), no. 1, 71-79.

[27] R. M. Wilson, Decomposition of complete graphs into subgraphs isomorphic to a given graph, Congressus Numerantium XV (1975), 647-659.

[28] A. Zak, Packing large trees of consecutive orders, arXiv:1510.07467 [math.CO] (2015). 